Proceedings of the Edinburgh Mathematical Society (2008) 51, 489-494 (C)

DOI:10.1017/S0013091506001180 Printed in the United Kingdom

\title{
PROPERTIES OF THE MAXIMAL OPERATORS ASSOCIATED WITH BASES OF RECTANGLES IN $\mathbb{R}^{3}$
}

\author{
ALEXANDER STOKOLOS \\ Department of Mathematics, DePaul University, \\ Chicago, IL 60614, USA (astokolo@math.depaul.edu)
}

(Received 21 September 2006)

\begin{abstract}
This paper is an attempt to understand a phenomenon of maximal operators associated with bases of three-dimensional rectangles of dimensions $(t, 1 / t, s)$ within a framework of more general Soria bases. The Jessen-Marcinkiewicz-Zygmund Theorem implies that the maximal operator associated with a Soria basis continuously maps $L \log ^{2} L$ into $L^{1, \infty}$. We give a simple geometric condition that guarantees that the $L \log ^{2} L$ class cannot be enlarged. The proof develops the author's methods applied previously in the two-dimensional case and is related to theorems of Córdoba, Soria and Fefferman and Pipher.
\end{abstract}

Keywords: maximal operators; differentiation bases; Soria bases

2000 Mathematics subject classification: Primary 42B25

In this paper we will deal with translation-invariant collections consisting of certain multidimensional rectangles (i.e. Cartesian products of one-dimensional intervals). We will call them bases. The most important result concerning a basis consisting of all the possible $n$-dimensional rectangles is the famous Jessen-Marcinkiewicz-Zygmund Theorem. The quantitative version of this theorem is this weak-type- $L\left(\log ^{+} L\right)^{n-1}$ estimate for the strong maximal operator

$$
M_{s} f(x)=\sup _{\text {all } R} \frac{1}{|R|} \int_{R \ni x}|f(y)| \mathrm{d} y: \quad\left|\left\{M_{s} f>\lambda\right\}\right| \leqslant C \int \frac{|f|}{\lambda}\left(1+\log ^{+} \frac{|f|}{\lambda}\right)^{n-1} .
$$

We say that a basis $\mathcal{B}$ is of weak type $L\left(\log ^{+} L\right)^{j}$ if condition (1) holds with $n-1$ replaced by $j$ and $M_{s} f$ replaced by

$$
M_{\mathcal{B}} f(x)=\sup _{R \in \mathcal{B}} \frac{1}{|R|} \int_{R \ni x}|f(y)| \mathrm{d} y .
$$

The estimate (1) was established by Fava [5] and, independently, by de Guzmán [3]. Testing the inequality on the characteristic function of the unit cube indicates that it is sharp. One can view this as testing the corresponding maximal operator on the $\delta$-function (for more discussion, see the 'final remarks' on p. 3240 of [6]). 
This estimate gets worse by a logarithmic factor with each increment of the dimension. On the other hand, Zygmund [9] demonstrated that the basis consisting of the Cartesian product of $k$-dimensional cubes and $n-k$ one-dimensional intervals is of weak type $L\left(\log ^{+} L\right)^{n-k}$. It would thus be natural to expect the properties of the bases to become worse with the addition of extra degrees of freedom. The converse could also be expected to be true: that the properties of the bases would become better with a reduction in the number of degrees of freedom. Zygmund has conjectured that if the basis consists of the $n$-dimensional rectangles whose side lengths are functions of the same $k$ independent variables $(k<n)$, then the basis should behave like the basis of all $k$-dimensional rectangles. For example, a basis of two-dimensional rectangles of side length $(t, \phi(t))$ with non-decreasing $\phi(t)$ is of weak type $(1,1)$. Córdoba [1] proved Zygmund's conjecture for an important particular case, establishing that the basis of three-dimensional rectangles of dimensions $(t, s, h(t, s))$ continuously maps $L \log ^{+} L$ into weak $L$ when $h(t, s)$ is a function that is non-decreasing in each variable.

Despite the progress that has been made towards the proof of the conjecture, it turns out that in stated generality it is false. Soria [7] has constructed a beautiful example of a basis of three-dimensional rectangles of dimensions $(t, t \phi(s), t \psi(s))$ with the nondecreasing functions $\phi(s)$ and $\psi(s)$, which has the same property as the basis of all possible three-dimensional rectangles.

Soria's result sheds light on the complexity of the three-dimensional case, while the two-dimensional case is now totally understood.

Let us be more specific. We call two rectangles $R$ and $R^{\prime}$ comparable and denote this by $R \sim R^{\prime}$ if there exists a translation placing one of them inside the other. In the opposite case we call them incomparable and write $R \nsim R^{\prime}$.

Now, for every rectangle $R \in \mathcal{B}$ we denote by $R^{*}$ the concentric rectangle of minimal measure containing $R$ that has side lengths of the form $2^{k}, k \in \mathbb{Z}$. Thus, to every basis $\mathcal{B}$ we attach, in a natural way, another basis $\mathcal{B}^{*}=\left\{R^{*} \mid R \in \mathcal{B}\right\}$ : a dyadic skeleton of the basis $\mathcal{B}$. It is clear that $\mathcal{B}$ and $\mathcal{B}^{*}$ have the same weak-type estimates.

The property

$$
\exists k>1 \quad \forall R_{1}, \ldots, R_{k} \in \mathcal{B}^{*} \quad \exists i \neq j, \quad R_{i} \sim R_{j}
$$

(henceforth called the 'w-property'), and an alternative one

$$
\forall k>1 \quad \exists R_{1}, \ldots, R_{k} \in \mathcal{B}^{*} \quad \forall i \neq j, \quad R_{i} \nsim R_{j}
$$

(henceforth called the 's-property'), were introduced in [8].

It turns out that in the two-dimensional case having the $s$-property makes a basis 'bad': any basis with this property behaves like the basis of all two-dimensional rectangles. On the other hand, a basis with the $w$-property is 'good' in the sense that it behaves like the basis of all squares (for more details see $[\mathbf{8}]$ ).

A model two-dimensional basis with the s-property is provided by the basis of rectangles of dimensions $(t, 1 / t)$. It is a weak-type- $L \log ^{+} L$ basis and this is the best possible estimate. The appearance of the logarithmic factor is a result of the integration of the $1 / t$ function. 
Switching to three-dimensional space and considering the basis of three-dimensional rectangles of dimensions $(t, 1 / t, s)$, one would expect the extra degree of freedom to bring an extra logarithm into the weak-type estimate. Surprisingly, this is not the case. Soria [7] noticed that this basis is a weak-type- $L \log ^{+} L$ basis too. This is because any two different rectangles from the $(t, 1 / t)$-basis are incomparable! Thus, the three-dimensional case turns out to be totally different from the two-dimensional case: both comparableness and incomparableness can improve the properties of bases.

The problem of investigating the three-dimensional case lies in the lack of covering methodology. The arsenal of available tools is very limited: there are only the standard covering arguments of Vitali (see, for example, [4]), Cordoba and Fefferman [2], Cordoba [1] and Fefferman and Pipher [6]. Further development of the general case seems quite difficult. It would, therefore, be natural to study important specific bases. One such example is a basis consisting of Cartesian products of the two-dimensional rectangles forming a certain basis $B$ in the $X Y$-plane (we will call this a projection basis) and arbitrary one-dimensional intervals in the $Z$-direction. We will call such bases Soria bases, because the $(t, 1 / t, s)$-basis is a model case. An understanding of the behaviour of these relatively simple bases would be a significant step forward in the understanding of the general situation.

There are examples of Soria bases of weak type $(1,1)$, of weak type $L \log ^{+} L$, and of weak type $L \log ^{2} L$. The purpose of this paper is to introduce a simple geometric condition on Soria bases that implies weak type $L \log ^{2} L$ (as opposed to, say, $L \log ^{+} L$ for the typical $(t, 1 / t, s)$ Soria basis). Note that the projection basis for $(t, 1 / t, s)$ is the $(t, 1 / t)$-basis, which consists of only incomparable rectangles; an intersection of any two such rectangles does not belong to the basis.

Behaviour opposite to this is specified by the following property:

$$
\forall k>1 \quad \exists R_{1}, \ldots, R_{k} \in B_{0}^{*} \quad \forall i \neq j, \quad\left(R_{i} \nsim R_{j}\right) \&\left(R_{i} \cap R_{j} \in B_{0}^{*}\right),
$$

which is henceforth referred to as the 'is-property'. Here $B_{0}^{*}$ denotes rectangles from $B^{*}$ with the left lower vertices at the origin.

Theorem. Let $\mathcal{B}$ be a Soria basis with the is-property. Then for any $0<\lambda<1$ there exists a set $E$ such that

$$
\left|\left\{M_{\mathcal{B}}\left(\chi_{E}\right)>\lambda\right\}\right| \geqslant C \int \frac{\chi_{E}}{\lambda} \log ^{2} \frac{\chi_{E}}{\lambda} \mathrm{d} x
$$

with some constant $C$ independent of $E$ and $\lambda$.

Proof. For a two-dimensional rectangle $R$ let $\operatorname{pr}_{x}(R)$ and $\operatorname{pr}_{y}(R)$ denote the projections of $R$ onto the $X$-axis and the $Y$-axis, respectively. The is-property implies that

$$
\begin{gathered}
\forall k \geqslant 1 \quad \exists R_{q}^{j} \in B^{*} \quad(1 \leqslant j \leqslant k, 1 \leqslant q \leqslant j), \\
R_{q}^{k} \nsim R_{p}^{k}, \quad 1 \leqslant q \neq p \leqslant k, \\
\operatorname{pr}_{x}\left(R_{q}^{j-1}\right)=\operatorname{pr}_{x}\left(R_{q}^{j}\right), \quad \operatorname{pr}_{y}\left(R_{q}^{j-1}\right)=\operatorname{pr}_{y}\left(R_{q+1}^{j}\right), \quad q=1, \ldots, j-1 .
\end{gathered}
$$



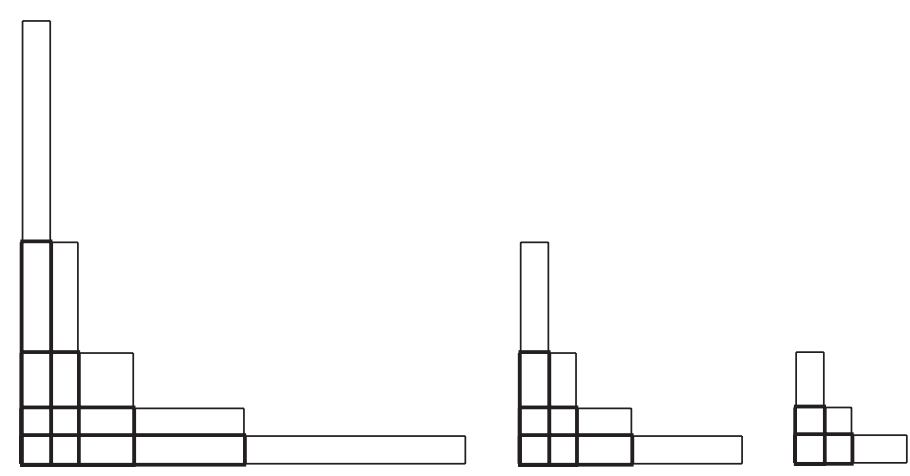

Figure 1. Projections $X_{5}, X_{4}, X_{3}, X_{2}$ and $X_{1}$.

Geometrically, this means that the stair-shaped set

$$
X_{j}=\bigcup_{1 \leqslant q \leqslant j} R_{q}^{j}
$$

contains contains a nested system of staircases, each of which is in turn a union of rectangles from $B^{*}$ (see Figure 1).

The characteristic feature of the pictures in Figure 1 is that each picture is an entire fragment of the previous one.

Now, for each rectangle $R_{i}^{j}$ let $H_{i}^{j}$ denote $\operatorname{pr}_{x}\left(R_{i}^{j}\right)$ and $V_{i}^{j}$ denote $\operatorname{pr}_{y}\left(R_{i}^{j}\right)$, i.e. $R_{i}^{j}=$ $H_{i}^{j} \times V_{i}^{j}$. Define the set $\Theta$ and the family of sets $Y^{k}$. Set

$$
\begin{aligned}
& \Theta^{1}=\left\{x_{1} \in H_{k}^{k}: \prod_{q=1}^{k-1} \sum_{s=0}^{2^{m_{q}-m_{k}-1}-1} \chi_{H_{q}^{k}}\left(x_{1}-2 s\left|H_{q}^{k}\right|\right)=1\right\}, \\
& \Theta^{2}=\left\{x_{2} \in V_{1}^{k}: \prod_{q=2}^{k} \sum_{s=0}^{2^{n_{q}-n_{1}-1}-1} \chi_{V_{q}^{k}}\left(x_{2}-2 s\left|V_{q}^{k}\right|\right)=1\right\},
\end{aligned}
$$

and $\Theta \equiv \Theta^{1} \times \Theta^{2}$.

From geometric reasoning, it is clear that $\left|\Theta^{1}\right|=2^{1-k}\left|H_{k}^{k}\right|$ and $\left|\Theta^{2}\right|=2^{1-k}\left|V_{1}^{k}\right|$, and so $|\Theta|=2^{2-2 k}\left|H_{k}^{k}\right|\left|V_{1}^{k}\right|$.

Next, for fixed $1 \leqslant i \leqslant j \leqslant k$ let $Y_{k}^{1, k}=H_{k}^{k}, Y_{1}^{2, k}=V_{1}^{k}$,

$$
\begin{aligned}
& Y_{i}^{1, j}=\left\{x_{1} \in H_{k}^{k}: \prod_{q=i}^{k-1} \sum_{s=0}^{2^{m_{q}-m_{k}-1}-1} \chi_{H_{q}^{k}}\left(x_{1}-2 s\left|H_{q}^{k}\right|\right)=1\right\}, \\
& Y_{i}^{2, j}=\left\{x_{2} \in V_{1}^{k}: \prod_{q=2}^{k-j+i 2^{n_{q}-n_{1}-1}-1} \sum_{s=0} \chi_{V_{q}^{k}}\left(x_{2}-2 s\left|V_{q}^{k}\right|\right)=1\right\}
\end{aligned}
$$

and $Y_{i}^{j} \equiv Y_{i}^{1, j} \times Y_{i}^{2, j}$. From geometric reasoning again,

$$
\left|Y_{i}^{1, j}\right|=2^{-(k-i)}\left|H_{k}^{k}\right|, \quad\left|Y_{i}^{2, j}\right|=2^{-(k-j+i-1)}\left|V_{1}^{k}\right|,
$$


and hence

$$
\left|Y_{i}^{j}\right|=2^{-2 k+j+1}\left|H_{k}^{k}\right|\left|V_{1}^{k}\right| \quad(1 \leqslant i \leqslant j \leqslant k) .
$$

Furthermore, from the definition of the sets $Y_{i}^{1, j}$ and $Y_{i}^{2, j}$ it follows that $Y_{i}^{1, j}$ is a union of translates of the intervals $H_{i}^{k}$, while $Y_{i}^{2, j}$ is a union of translates of the intervals $V_{k-j+i}^{k}$. By the assumptions of the theorem,

$$
H_{i}^{k}=H_{i}^{k-1}=\cdots=H_{i}^{j+1}=H_{i}^{j}
$$

and

$$
V_{q}^{k}=V_{q-1}^{k-1}=\cdots=V_{q-(k-j)}^{j}
$$

which upon substituting $k-j+i$ for $q$ yields

$$
V_{k-j+i}^{k}=V_{i}^{j}
$$

Thus, $Y_{i}^{1, j}$ consists of translated intervals $H_{i}^{j}$, and $Y_{i}^{2, j}$ consists of translated intervals $V_{i}^{j}$. Consequently, $Y_{i}^{j}$ consists of translated rectangles $R_{i}^{j}$, i.e. for every $\left(x_{1}, x_{2}\right) \in Y_{i}^{j}$ there is a translation $\tau$ such that $\tau\left(R_{i}^{j}\right) \ni\left(x_{1}, x_{2}\right)$. Similarity considerations show that

$$
\frac{\left|\tau\left(R_{i}^{j}\right) \cap \Theta\right|}{\left|\tau\left(R_{i}^{j}\right)\right|}=\frac{\left|R_{i}^{j} \cap \Theta\right|}{\left|R_{i}^{j}\right|}=\frac{\left|Y_{i}^{j} \cap \Theta\right|}{\left|Y_{i}^{j}\right|} .
$$

Furthermore, the definitions of $\Theta^{1}$ and $\Theta^{2}$ imply that

$$
\Theta^{1} \subset Y_{i}^{1, j}, \quad \Theta^{2} \subset Y_{i}^{2, j}
$$

and hence $\Theta \subset Y_{i}^{j}$ and

$$
\frac{\left|Y_{i}^{j} \cap \Theta\right|}{\left|Y_{i}^{j}\right|}=\frac{|\Theta|}{\left|Y_{i}^{j}\right|}=2^{1-j} \quad(1 \leqslant i \leqslant j \leqslant k)
$$

Now set

$$
U \equiv \Theta \times[0,1], \quad Z_{i}^{j} \equiv Y_{i}^{j} \times\left[0,2^{k-j}\right], \quad I_{i}^{j} \equiv R_{i}^{j} \times\left[0,2^{k-j}\right] \quad(1 \leqslant i \leqslant j \leqslant k) .
$$

Obviously, the $I_{i}^{j}$ are pairwise-incomparable three-dimensional rectangles with dyadic side lengths, so if we set

$$
W=\bigcup_{j=1}^{k} \bigcup_{i=1}^{j} Z_{i}^{j}
$$

then

$$
|W| \sim \sum_{j=1}^{k} \sum_{i=1}^{j}\left|Z_{i}^{j}\right|=\sum_{j=1}^{k} \sum_{i=1}^{j} 2^{k-j}\left|Y_{i}^{j}\right|=\sum_{j=1}^{k} \sum_{i=1}^{j} 2^{k-j} 2^{j-1}|\Theta|=2^{k-2} k(k+1)|U| .
$$


By the above considerations, for every $\left(x_{1}, x_{2}, x_{3}\right) \in W$ there is a rectangle $I_{i}^{j}$ and a translation $\bar{\tau}$ such that $\left(x_{1}, x_{2}, x_{3}\right) \in \bar{\tau}\left(I_{i}^{j}\right)$ and

$$
\bar{\tau}\left(I_{i}^{j}\right)=\tau\left(R_{i}^{j}\right) \times\left[0,2^{k-j}\right],
$$

where $\tau$ is as defined above. Hence,

$$
\frac{\left|\bar{\tau}\left(I_{i}^{j}\right) \cap U\right|}{\left|\bar{\tau}\left(I_{i}^{j}\right)\right|}=\frac{\left|\tau\left(R_{i}^{j}\right) \cap \Theta\right|}{2^{k-j}\left|\tau\left(R_{i}^{j}\right)\right|}=\frac{\left|R_{i}^{j} \cap \Theta\right|}{2^{k-j}\left|R_{i}^{j}\right|}=\frac{2^{1-j}}{2^{k-j}}=2^{1-k} .
$$

These estimates show that

$$
\begin{gathered}
W \subset\left\{x: M_{B^{*}}\left(\chi_{U}\right)(x) \geqslant 2^{1-k}\right\}, \\
\left|\left\{x: M_{B^{*}}\left(\chi_{U}\right)(x) \geqslant 2^{1-k}\right\}\right| \geqslant|W| \geqslant k^{2} 2^{k-2}|U| \geqslant \frac{1}{2} \int \frac{\chi_{U}}{2^{1-k}} \log ^{2} \frac{\chi_{U}}{2^{1-k}} \mathrm{~d} x,
\end{gathered}
$$

which can be seen as the reverse to the Jessen-Marcinkiewicz-Zygmund $L \log ^{2} L$ estimate.

This completes the proof of the theorem.

Acknowledgements. The author's research was partially supported by the DePaul University Leave Program. The author is grateful to Marshall Ash for useful discussions and for help in the preparation of the manuscript.

\section{References}

1. A. Córdoba, Maximal functions, covering lemmas and Fourier multipliers, in Harmonic Analysis in Euclidean Spaces, Part 1, Williamstown, MA, 1978, Proceedings of Symposia in Pure Mathematics, Volume 35, pp. 29-49 (American Mathematical Society, Providence, RI, 1979).

2. A. Córdoba And R. Fefferman, A geometric proof of the strong maximal theorem, Annals Math. 102 (1975), 95-100.

3. M. DE Guzmán, An inequality for the Hardy-Littlewood maximal operator with respect to a product of differentiation bases, Studia Math. 49 (1973), 185-194.

4. M. DE GuzMán, Differentiation of integrals in $R^{n}$, Lecture Notes in Mathematics, Volume 481 (Springer, 1975).

5. N. FAVA, Weak type inequalities for product operators, Studia Math. 42 (1972), 271-288.

6. R. Fefferman and J. Pipher, A covering lemma for rectangles in $\mathbb{R}^{n}$, Proc. Am. Math. Soc. 133 (2005), 3235-3241.

7. F. SORIA, Examples and counterexamples to a conjecture in the theory of differentiation of integrals, Annals Math. 123 (1986), 1-9.

8. A. M. Stokolos, Zygmund's program—some partial solutions, Annales Inst. Fourier $\mathbf{5 5}$ (2005), 1439-1453.

9. A. Zygmund, A note on the differentiability of multiple integrals, Colloq. Math. 16 (1967), 199-204. 